\title{
BMJ Open Antibiotic prescribing in long-term care facilities: a qualitative, multidisciplinary investigation
}

\author{
Aoife Fleming, ${ }^{1}$ Colin Bradley, ${ }^{2}$ Shane Cullinan, ${ }^{1}$ Stephen Byrne ${ }^{1}$
}

To cite: Fleming A, Bradley C, Cullinan $\mathrm{S}$, et al. Antibiotic prescribing in long-term care facilities: a qualitative, multidisciplinary investigation. BMJ Open 2014;4:e006442. doi:10.1136/bmjopen-2014006442

- Prepublication history and additional material is available. To view please visit the journal (http://dx.doi.org/ 10.1136/bmjopen-2014006442).

Received 21 August 2014 Revised 8 October 2014 Accepted 17 October 2014

CrossMark

${ }^{1}$ Pharmaceutical Care Research Group, School of Pharmacy, University College Cork, Ireland

${ }^{2}$ Department of General Practice, University College Cork, Ireland

Correspondence to

Aoife Fleming;

a.fleming@ucc.ie

\section{ABSTRACT}

Objectives: To explore healthcare professionals' views of antibiotic prescribing in long-term care facilities (LTCFs). To use the findings to recommend intervention strategies for antimicrobial stewardship in LTCFs.

Design: Qualitative semistructured interviews were conducted. The data were analysed by thematic content analysis. After the interviews, the emerging findings were mapped to the theoretical domains framework (TDF), and the behaviour change wheel and behaviour change technique (BCT) taxonomy were used to recommend future intervention strategies.

Participants: Interviews were conducted with 37 healthcare professionals who work in LTCFs (10 general practitioners, 4 consultants, 14 nurses, 9 pharmacists) between December 2012 and March 2013.

Setting: Interviews were conducted in the greater Cork region.

Results: The main domains from the TDF which emerged were: 'Knowledge', 'Environmental context and resources', 'Social influences', 'Beliefs about consequences', 'Memory, attention and decision making', with the findings identifying a need for 'Behavioural regulation'. Many participants believed that antibiotic prescribing was satisfactory at their LTCF, despite the lack of surveillance activities.

Conclusions: This study, using the TDF and BCT taxonomy, has found that antibiotic prescribing in LTCFs is influenced by many social and contextual factors. The challenges of the setting and patient population, the belief about consequences to the patient, and the lack of implementation of guidelines and knowledge regarding antibiotic prescribing patterns are significant challenges to address. On the basis of the study findings and the application of the TDF and BCT taxonomy, we suggest some practical intervention functions for antimicrobial stewardship in LTCFs.

\section{INTRODUCTION}

Antibiotic use in long-term care facilities (LTCFs) contributes to the emergence of multidrug-resistant pathogens and healthcareacquired infections. ${ }^{1}$ The Royal College of Physicians in Ireland Policy Group on Health Care Associated Infection in Nursing Homes recommends that implementation of best

\section{Strengths and limitations of this study}

This study is the first to undertake qualitative interviews investigating antibiotic prescribing in long-term care facilities (LTCFs) and to map the findings to the theoretical domains framework, capability, opportunity, motivation and behaviour model and behaviour change technique taxonomy in order to recommend intervention strategies.

- The study captures the views of the key healthcare professionals involved in antibiotic prescribing in LTCFs, general practitioners, consultants, nurses and pharmacists.

- The findings indicate that antibiotic prescribing in LTCFs is strongly influenced by the context of healthcare delivery in LTCFs. There is a need for 'Behavioural regulation' strategies such as antibiotic surveillance in LTCFs, and intervention functions such as setting goals, education, audit, feedback and monitoring may contribute to improved antimicrobial stewardship in LTCFs.

- All the participants in the study were based in the same region in Ireland and may hold different views from those in other countries or regions. However, the broad sample and depth of discussion offers valuable insights into the Irish LTCFs context.

practice for antibiotic stewardship in LTCFs and ongoing research to guide interventions is necessary. $^{2}$ In the Irish context, the healthcare associated infections in long-term care (HALT) point prevalence studies have reported a higher prevalence $(10 \%)$ of antibiotic prescribing compared to the European average $(5 \%)$ in 2010 and $2013 .^{3}$ Internationally, studies have suggested that between $25 \%$ and $75 \%$ of antibiotic prescriptions in LTCFs are inappropriate and that antimicrobial resistance (AMR) is rising. ${ }^{1} 4$ Quantitative studies investigating antibiotic prescribing in LTCFs have suggested that prescribing patterns are driven by prescriber factors rather than infection prevalence or antimicrobial stewardship initiatives. ${ }^{5}$ It is necessary to investigate the factors that influence antibiotic 
prescribing behaviours in LTCFs. In order to capture this information, the views of the healthcare professionals that are central to this process must be explored. Recent systematic reviews of qualitative studies of antibiotic prescribing behaviour have focused mainly on the overall primary care or secondary care setting without focusing on the LTCFs setting specifically. ${ }^{6}$ It is necessary to evaluate LTCFs as a separate setting for antibiotic prescribing because patient care is often influenced by factors unique to this setting, such as the comorbidities of the patient population and organisational culture. ${ }^{8}$

The use of theory to understand the mechanisms of action of intervention strategies to change behaviour has been shown to improve the effectiveness of interventions. ${ }^{9}$ In recent years, the theoretical domains framework (TDF) has gained much attention as a potentially overarching theoretical framework to identify the areas where behavioural change interventions can focus. ${ }^{10}$ The TDF was initially developed in response to requests from implementation researchers who recognised the need for an integrative framework to address the behaviour change factors relevant to intervention studies. ${ }^{11}$ It has been used in many different types of studies and the framework has been refined and validated. ${ }^{12}$ It consists of 14 domains which consist of 84 component constructs. ${ }^{12}$ The framework comprehensively draws together, from 33 theories of behaviour, the crucial influences on behaviour. ${ }^{10}$ The TDF domains are presented in table 1 with a sample construct. The TDF has been used in qualitative studies to guide the development of interview topic guides and it has also been used as a coding framework in the analysis of qualitative material. ${ }^{10} 13$ Researchers in this area have designed a behaviour change wheel which consists of capability, opportunity, motivation and behaviour components or the COM-B model as it is also known (figure 1). ${ }^{11}{ }^{14}$ The corresponding behaviour change technique taxonomy (BCT taxonomy) has been developed in order to standardise the content and reporting of intervention studies. ${ }^{11} 14{ }^{15}$ In previous qualitative studies of antibiotic prescribing in LTCFs, a behavioural theory has not been used to inform the evaluation or to identify areas for antimicrobial stewardship. ${ }^{16}{ }^{17}$ In order to fully capture and understand the factors influencing antibiotic prescribing, the views of all healthcare professionals involved in this process is required. The advantage of conducting qualitative investigations before the implementation of an intervention is that the findings can inform the content and delivery of the intervention based on healthcare professional views and experiences. ${ }^{18}$

With increasing rates of AMR and higher than average rates of antibiotic prescribing in Irish LTCFs, an in-depth qualitative investigation of the views of all key healthcare professionals involved in this process is

Table 1 Theoretical domains presented with explanatory definition and sample construct

\begin{tabular}{|c|c|}
\hline Domain & Definition and example of a construct \\
\hline Knowledge & An awareness of the existence of something, for example, procedural knowledge \\
\hline Skill & An ability or proficiency acquired through practice, for example, competence \\
\hline $\begin{array}{l}\text { Social/professional role and } \\
\text { identity }\end{array}$ & $\begin{array}{l}\text { A coherent set of behaviours and displayed personal qualities of an individual in a social } \\
\text { or work setting, for example, professional confidence }\end{array}$ \\
\hline Beliefs about capabilities & $\begin{array}{l}\text { Acceptance of the truth, reality or validity about an ability, talent or facility that a person } \\
\text { can put to constructive use, for example, self-confidence }\end{array}$ \\
\hline Optimism & $\begin{array}{l}\text { The confidence that things will happen for the best or that desired goals will be attained, } \\
\text { for example, optimism, pessimism }\end{array}$ \\
\hline Beliefs about consequences & $\begin{array}{l}\text { Acceptance of the truth, reality or validity about outcomes of a behaviour in a given } \\
\text { situation, for example, outcome expectancies }\end{array}$ \\
\hline Reinforcement & $\begin{array}{l}\text { Increasing the probability of a response by arranging a dependent relationship, or } \\
\text { contingency, between the response and a given stimulus, for example, rewards }\end{array}$ \\
\hline Intentions & $\begin{array}{l}\text { A conscious decision to perform a behaviour or resolve to act in a certain way, for } \\
\text { example, stability of intentions }\end{array}$ \\
\hline Goals & $\begin{array}{l}\text { Mental representations of outcomes or end states that an individual wants to achieve, for } \\
\text { example, goal/target setting }\end{array}$ \\
\hline $\begin{array}{l}\text { Memory, attention and decision } \\
\text { processes }\end{array}$ & $\begin{array}{l}\text { The ability to retain information, focus selectively on aspects of the environment and } \\
\text { choose between two or more alternatives, for example, decision-making }\end{array}$ \\
\hline $\begin{array}{l}\text { Environmental context and } \\
\text { resources }\end{array}$ & $\begin{array}{l}\text { Any circumstances of a person's situation or environment that discourages or encourages } \\
\text { the development of skills and abilities, independence, social competence and adaptive } \\
\text { behavior, for example, resources }\end{array}$ \\
\hline Social influences & $\begin{array}{l}\text { Those interpersonal processes that can cause individuals to change their thoughts, } \\
\text { feelings or behaviours, for example, social pressure }\end{array}$ \\
\hline Emotion & $\begin{array}{l}\text { A complex reaction pattern, involving experiential, behavioural and physiological elements, } \\
\text { by which the individual attempts to deal with a personally significant matter or event, for } \\
\text { example, anxiety }\end{array}$ \\
\hline Behavioural regulation & $\begin{array}{l}\text { Anything aimed at managing or changing objectively observed or measured actions, for } \\
\text { example, self-monitoring }\end{array}$ \\
\hline
\end{tabular}


Figure 1 The behaviour change wheel. $^{11}$
Sources of behaviour

Intervention functions

Policy categories

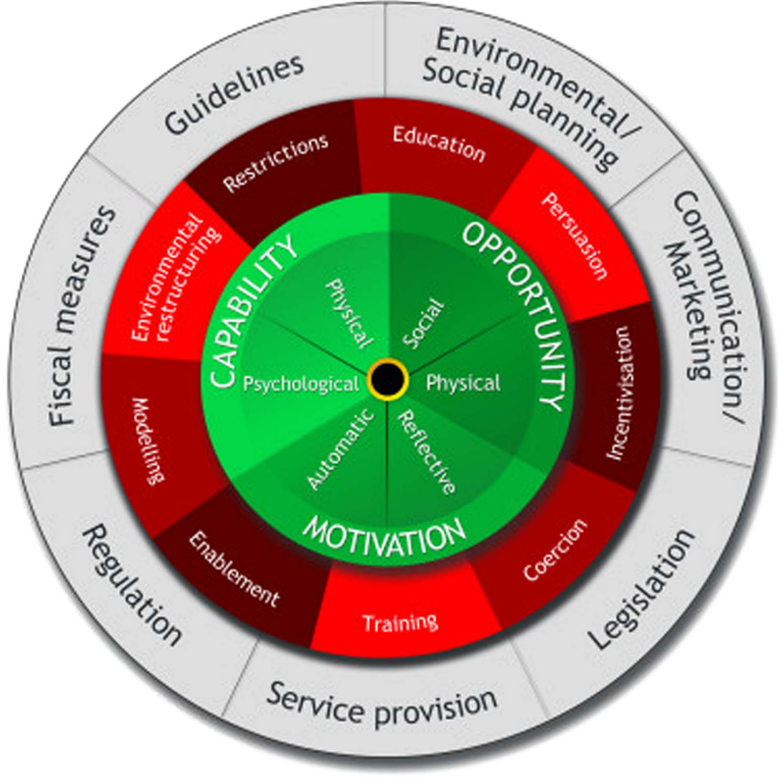

summarised in table 2. The topic guide was made relevant to the appropriate healthcare professional group in terms of the question perspective, but the key issues were the same across the board. The domains of the TDF were considered when designing the topic guide, but the structure was not restricted by the TDF at this stage to allow for the emergence of unanticipated and unprompted issues during the interviews. ${ }^{19}$ The topic guide was refined after being piloted by interviewing one pharmacist and two GPs. Only one of the pilot GP transcripts is included in the final analysis.

Table 2 Summary of the interview topic guide

\begin{tabular}{ll}
\hline Area & Issues discussed \\
\hline Demographic information & $\begin{array}{l}\text { Years in practice, years } \\
\text { working in a LTCF }\end{array}$ \\
Process and & Procedure for diagnosing \\
tecision-making & Challenges in treating infection \\
& Involvement with other \\
& healthcare professionals \\
& Use of or awareness of a \\
& guideline for antibiotic \\
Knowledge & prescribing \\
& Antibiotics commonly \\
& prescribed \\
& Knowledge of local \\
& antimicrobial resistance \\
& patterns \\
& Consequences of not \\
& prescribing antibiotics \\
& Problems associated with \\
& antibiotics \\
& Current activities, audits or \\
& prescribing feedback \\
& Areas where more support is \\
needed
\end{tabular}


The one-to-one interviews were conducted by $\mathrm{AF}$ at the participant's place of work (LTCFs, GP surgeries, consultants' offices, community pharmacies), in a quiet room to maintain privacy and confidentiality, at a date and time convenient for them. The purpose of the study was outlined to participants. The interviewer (AF) presented herself as a researcher and did not engage in discussion with the participants about the study or topic guide before the interview so as not to bias their feedback. Some demographic information was collected. The interviews were audio recorded, with participant approval and written informed consent, and the participants were encouraged to think of specific case examples to elaborate on the topics. The interviewer prompted and explored issues in more detail as appropriate. ${ }^{19}$ The interview allowed for the emergence of unprompted information and themes. All interviews were anonymised and transcribed by $\mathrm{AF}$ and preliminary familiarisation was begun during the transcription process. In this way, data analysis began at an early stage and the topic guide was constantly reviewed and new topics were introduced throughout the interview process as needed. The interview transcript was available to the participants on request. Field notes were recorded after each interview.

\section{Analysis}

As described above, an iterative process of data collection and analysis was conducted. All transcripts were coded in QSR Internationals NVivo Qualitative Data Analysis Software V.10. ${ }^{22}$ The initial phase of familiarisation involved several readings of the interview transcripts. The transcripts were initially coded by AF and a coding scheme was developed. On the basis of the initial familiarisation, we decided not to analyse the interviews in three separate healthcare professional groups. This decision was taken as the topic guide was similar between the groups and similar issues, from different groups, were emerging throughout all interviews. To ensure consistencies in coding, three coders (AF, SC and SB) independently coded four interview transcripts. The inter-rater reliability was high and any disagreements were resolved by discussion. The participant's own language was often used in the naming of codes in order to maintain a faithful representation of their opinions and experiences. The codes or specific beliefs were then attributed to the domains of the TDF. The next stage involved identifying what behaviours needed to change and what methods could be recommended to achieve this. This was conducted by mapping the TDF domains to the behaviour change wheel, specifically the capability, opportunity and motivation components. ${ }^{11}$ The appropriate BCT taxonomy (V.1) was applied to suggest intervention functions for antimicrobial stewardship in LTCFs. A completed checklist of the consolidated criteria for reporting qualitative research (COREQ) is presented in online supplementary material table A. ${ }^{23}$

\section{RESULTS}

Thirty-seven interviews were conducted in total (14 doctors (10 GPs, 4 consultants), 14 nurses and 9 pharmacists) from a range of LTCF settings. Participant detail is provided in table 2. The interviews ranged from $10 \mathrm{~min}$ to $35 \mathrm{~min}$ (mean interview length $22 \mathrm{~min}$ ). The key themes are presented by means of the relevant domain from the TDF. Participant quotes are represented by profession (general practitioner $=\mathrm{GP}$, consultant $=\mathrm{C}$, nurse $=\mathrm{N}$ and pharmacist $=\mathrm{P}$ ) and the corresponding number refers to their details in table 3 .

\section{THEORETICAL DOMAINS FRAMEWORK}

The analysis identified key domains of the TDF that were found to be relevant and they are described below. The other domains that were not identified (optimism, reinforcement, intentions, goals and emotions) are not discussed as not enough references to the relevant constructs were made.

\section{Knowledge}

It was decided to merge the domains 'Knowledge' and 'Skill' as the constructs emerging were overlapping and most findings related to the knowledge factors. The participants did not report that the challenge in diagnosing and treating patients in an LTCF was due to a lack of skills or need for further training in undertaking physical tasks. Knowledge of antibiotic guidelines was variable among all participants. Many participants, from all professions, were not aware of the Guidelines for Antimicrobial Prescribing in Primary Care 2011 or of the Health Protection Surveillance Centre (HPSC) guidelines for the management of catheter-related and non-catheter-related urinary tract infections. ${ }^{24}{ }^{25}$ In most cases, participants report a passive, rather than active, disregard of guidelines. Nurses in the public setting were more aware of HPSC guidelines but reported that they are not often adhered to.

so we have all the antibiotic guidelines, we have the primary care guidelines for antibiotic prescribing which don't give you exact antibiotics to prescribe. Like generally we use the CUH [Cork University Hospital], MUH [Mercy University Hospital] ones do you know those antimicrobial guidelines. (GP2)

The interpretation of urine samples from catheterised patients poses a challenge in LTCFs. Asymptomatic bacteriuria is an area where doctors and nurses felt unsure about whether to prescribe antibiotics or not. Several consultants identified that this is often an area of antibiotic overuse.

That is a big bug bear of mine, the UTI, the old person with a UTI, it drives me crazy. Every old person has a UTI and I'd say at least once a day I say to somebody 'you know if you take a room full of frail old people half of them will have dirty urine, it doesn't mean they have a UTI'. (C2) (UTI=Urinary Tract Infection) 
Table 3 List of participants interviewed, years of experience and LTCF setting

\begin{tabular}{|c|c|c|c|c|}
\hline $\begin{array}{l}\text { Doctor (general } \\
\text { practitioners) }\end{array}$ & Gender & $\begin{array}{l}\text { Years of medical } \\
\text { experience }\end{array}$ & $\begin{array}{l}\text { LTCF category and bed } \\
\text { occupancy }\end{array}$ & $\begin{array}{l}\text { Years of experience in } \\
\text { an LTCF }\end{array}$ \\
\hline 1. & $\mathrm{~F}$ & 15 & Private (12 patients in an LTCF) & 15 \\
\hline 2. & $\mathrm{~F}$ & 15 & Public/private (a 63 bed LTCF) & 8 \\
\hline 3. & $\mathrm{~F}$ & 2 & 1 Public, 1 Private & 2 \\
\hline 4. & M & 9 & Private (14 patients in an LTCF) & 9 \\
\hline 5. & $\mathrm{~F}$ & 10 & $\begin{array}{l}\text { Private ( } 15 \text { patients in an LTCF) } \\
\text { Public ( } 20 \text { patients in an LTCF) }\end{array}$ & 8 \\
\hline 6. & M & 1 & Private (10 patients in an LTCF) & 1.5 \\
\hline 7. & M & $>20$ & Private $(>100$ in total) & $>20$ \\
\hline 8. & M & 19 & Voluntary (>100 in total) & 19 \\
\hline 9. & $M$ & $>30$ & $\begin{array}{l}\text { Mixed setting (patient number } \\
\text { varies) }\end{array}$ & $>30$ \\
\hline 10. & $M$ & 5 & $\begin{array}{l}\text { Mixed setting (patient number } \\
\text { varies) }\end{array}$ & 5 \\
\hline
\end{tabular}

\begin{tabular}{|c|c|c|c|c|}
\hline Doctor (consultant) & Gender & Specialty & LTCF category & $\begin{array}{l}\text { Years of experience in } \\
\text { an LTCF }\end{array}$ \\
\hline 1. & $\mathrm{M}$ & Geriatrician & Public, voluntary & $>5$ \\
\hline 2. & M & Geriatrician & Public & $>5$ \\
\hline 3. & $\mathrm{~F}$ & Geriatrician & Public & $>5$ \\
\hline 4. & $M$ & Microbiologist & Public, private, voluntary & $>5$ \\
\hline Pharmacist & Gender & Years of experience & $\begin{array}{l}\text { LTCF category (range bed } \\
\text { occupancy) }\end{array}$ & $\begin{array}{l}\text { Years of experience in } \\
\text { an LTCF }\end{array}$ \\
\hline 1. & $\mathrm{M}$ & 15 & Public, (48) & 5 \\
\hline 2. & $\mathrm{~F}$ & 8 & Public and Private (13-250) & 8 \\
\hline 3. & $\mathrm{~F}$ & 30 & Private $(40-120)$ & 7 \\
\hline 4. & $\mathrm{~F}$ & 5 & Private $(40-120)$ & $<1$ \\
\hline 5. & M & 35 & Private $(50-60)$ & 20 \\
\hline 6. & $\mathrm{~F}$ & 1 & Private (25) & 1 \\
\hline 7. & M & 14 & Private (25) & 14 \\
\hline 8. & $\mathrm{~F}$ & 18 & Public (>150) & 2 \\
\hline 9. & $M$ & 15 & Public (38) & 5 \\
\hline Nurse & Gender & $\begin{array}{l}\text { Years of nursing } \\
\text { experience }\end{array}$ & LTCF category & $\begin{array}{l}\text { Years of experience in } \\
\text { an LTCF }\end{array}$ \\
\hline 1. Staff nurse & $\mathrm{F}$ & 21 & Private (50) & 4 \\
\hline 2. Staff nurse & $\mathrm{F}$ & 10 & Private (50) & 4 \\
\hline 3. CNM & $\mathrm{F}$ & 25 & Public (40) & 11 \\
\hline 4. Staff nurse & $\mathrm{F}$ & 16 & Public (40) & 5 \\
\hline 5. CNM & $\mathrm{F}$ & 15 & Public (38) & 12 \\
\hline $\begin{array}{l}\text { 6. Advanced nurse } \\
\text { practitioner and nurse } \\
\text { prescriber }\end{array}$ & $\mathrm{F}$ & 26 & Public $(>100)$ & 19 \\
\hline 7. CNM & $\mathrm{F}$ & 41 & Voluntary $(>100)$ & 6 \\
\hline 8. Staff nurse & $\mathrm{F}$ & 30 & Voluntary (30) & 30 \\
\hline 9. Staff nurse & $\mathrm{F}$ & 11 & Public (38) & 11 \\
\hline $\begin{array}{l}\text { 10. CNM and nurse } \\
\text { prescriber }\end{array}$ & $\mathrm{F}$ & 33 & Public (38) & 26 \\
\hline 11. CNM & $\mathrm{F}$ & 32 & Public/Private (60) & 20 \\
\hline 12. Staff nurse & $\mathrm{F}$ & 11 & Public/private (60) & 3 \\
\hline 13. IPCN & $\mathrm{F}$ & 15 & Public (multisite) & 10 \\
\hline 14. IPCN & $\mathrm{F}$ & 15 & Public (multisite) & 10 \\
\hline
\end{tabular}

Many participants conveyed confidence in their clinical knowledge due to their years of experience in LTCF practice and their in-depth knowledge of the individual patients.
So it can be very difficult to know, you are going by a bit of guesswork, a bit of analysis of results, a bit of examination, a bit of the history from the nurse, it depends on how well the nurse knows them as well, how changed 
they are from their usual baseline. Then you make a decision. You probably have a lower threshold for using antibiotics in long term care facilities because of all of those factors. (GP9)

In terms of clinical knowledge of the different antibiotics and their indications, it was evident that this varies between participants. Detailed knowledge of antibiotic microbiological coverage or recommended infection indication was not displayed or reported in the findings. Participants were more likely to refer to 'strong antibiotics' and only rare references to 'first line antibiotics' were made. All participants are aware that AMR is a growing public health problem and that overuse of antibiotics is a contributory factor, but few had any insight into local AMR data or referred to AMR as a serious problem in their LTCF.

... we only have a problem with resistance when it comes to urine infections because that is where I think we are over-treating. (GP4)

Closely aligned to 'Knowledge' findings are the themes from the 'Belief of capabilities' domain. Nurses expressed confidence in providing a high quality of care for the patients and that the more qualified nurses, nurse prescribers and clinical nurse managers provide valuable support to all nurses.

...she is very good (nurse manager) and very with it and she links up with the doctors quite a bit. If she has an issue they really listen to her as well. (N4)

Doctors also expressed professional confidence in caring for LTCF patients with infection. Guidelines are seen as a useful reference, but deviations from the guidelines were justified by relying on their own, or on the nurse's, clinical judgement and expertise.

nothing in medicine is black and white so you can't have guidelines, guidelines are just that, they are guidelines not protocols. I mean that is the difference people need to understand, protocols are something you have to stick to. (GP4)

I have no issue with guidelines you know. I think the most important thing is that when veering away from guidelines is justifying what we are doing. (GP7)

You don't want them (nurses) to see somebody in the bed who they are worried about and say oh she's not ticking such a box so I'm not going to ask the doctor to see her. (GP1)

The Pharmacists interviewed expressed confidence in the medicines management service they provide to the LTCFs but are less empowered in terms of expanding their clinical role. The reasons for this are the lack of time and the need for further training and guidelines in this area.
I think if they had more structured antibiotic CPD for antibiotics in nursing homes and even for pharmacies if we had more specific stuff it would be a big help. (P4). (CPD=Continuing Professional Development)

I would be fairly confident but sure I have all the resources here so I can have a quick look and go through them, I wouldn't know all of it off the top of my head, some of it I would. (P9)

I would like to be involved in some sort of you know developing some sort of protocol or guidelines within the nursing home, provided we are given the resources and the time to do that with a multidisciplinary team.... (P2)

\section{Social/professional role and identity}

The responsibility for antibiotic prescribing was clearly assigned to the doctor, but interestingly the key role of the nurse in that process was also conveyed by all professions.

I would be the one dealing with the GPs all the time on their rounds...so even though I think so and so might need an antibiotic or whatever, it is the doctors call in the end. (N3)

the way we operate is it is a nursing led facility and we come in to support that nursing lead. We are very lucky with the level of clinical nurse specialists that are there. So they have that higher training in dealing with elderly people, so they provide the care effectively and they rope us in then if there are issues that they are unhappy with or if there are issues as regards to prescribing. So we get involved if they have a concern about a patient or regarding a possibility or probability of infection. (GP7)

It was reported that between doctors, antibiotic prescribing practices vary in terms of the volume and choice of antibiotics prescribed. It was reported several times that out-of-hours doctors are often more likely to prescribe antibiotics. The main reason to explain this is that the patient is generally sicker if a doctor has to visit out of hours and an antibiotic is prescribed to avoid hospitalisation or a revisit.

you know if they are calling SouthDoc the patient has a fever, clinical signs, a bad cough, you are probably more likely to prescribe than not. (GP9)

(SouthDoc is the out-of-hours doctors service in the greater Cork region)

The difficulties, as reported by an out-of-hours doctor, are that they do not know the patient's medical history, they have limited diagnostic equipment, the patient is often very ill and they may be under pressure from the patient's family or nurse to prescribe. Some GPs reported that out-of-hours doctors may not prescribe first-line antibiotics.

...you are called as an out of hours doctor you often times have little option but to prescribe an antibiotic 
because you don't know the patient, you don't know the staff, you often don't know the background and you may not have complete notes in the history. (GP5)

The role of the pharmacist in antimicrobial stewardship has not developed considerably in LTCFs based on the reports of those interviewed. Some reported that they already attend clinical multidisciplinary meetings with the GPs and nurses and would welcome the opportunity to engage in this further, with support and appropriate training to improve knowledge. Others referred to the lack of time to engage in antimicrobial stewardship and that the priorities for pharmacists in LTCFs were other medicines management issues.

In terms of antibiotics I don't know necessarily if there is a huge role there, there are roles in other medicines management issues but not particularly antibiotics. (P9)

\section{Social influences}

The social context within which antibiotics are prescribed in LTCFs is clearly evident in the findings. The influence of nurses on doctors' decisions when managing patients with infection, especially when the decision to prescribe an antibiotic or not is made, was referred to frequently and by all groups of professionals. The nurses act in a gatekeeper role by communicating patient care issues and organising clinical assessments by the doctor when they visit the LTCF. In some cases, their influence in the decision-making process of whether to prescribe an antibiotic can be felt as a pressure by doctors.

...they certainly guide us in our prescribing, they are probably, I don't know is this a fair or unfair thing to say but they are probably happier when we prescribe because at least they know something has been treated. (GP2)

You sometimes feel that you do come under pressure to prescribe, and you have to sort of avoid that you know. (GP8)

Sometimes the doctors are guided by what we would suggest and what we feel or think. I suppose they just kind of, they are of the opinion we are with the patient so much more than they are but some doctors, definitely not all of them, some of them would defer to the nurse a little bit. (N9)

On the other hand, some nurses also discussed their influence on doctors in terms of delaying antibiotic prescriptions by suggesting 'watchful waiting' or that the doctor would reassess the patient in a few days and reconsider the need for an antibiotic at that point.

The role of the pharmacist is mainly in screening for drug interactions and providing medicines information, rather than influencing the antibiotic prescribing process. The influence of the resident's family on doctors and nurses to assess their relative occurs but was not linked to a pressure to prescribe an antibiotic. Families tend to be satisfied once the doctor has made a clinical assessment, even if they do not prescribe an antibiotic. The importance of including the families in the decision-making process and establishing goals of care for patients was underlined by many doctors, and interestingly by all the consultant geriatricians.

...the family would be insistent on them being seen by a doctor most of time and influence the nurse to call you but once you come and see them and assess them, no it would be uncommon that they would insist on an antibiotic. (GP9)

My feeling about prophylactic antibiotics for UTIs and stuff is I ask the family and the patient 'do you feel it is helping or making a difference' and if it isn't I stop it. (C2)

\section{Environmental context and resources}

The key contextual issue raised is that the management of infection in LTCFs is complicated by a high level of comorbidity, cognitive impairment and dementia in these patients. The lack of diagnostic equipment and interpretation of microbiology results is a significant challenge for doctors and nurses. They also reported that these elderly patients do not always have a high temperature on infection and are often not able to communicate their symptoms. This links to 'Social Influences' as doctors depend greatly on the nurse's support to detect the patient's signs of infection. The restricted access to a doctor was also a challenge to this process as many LTCFs do not have an on-site medical officer but receive care from GPs who visit infrequently or only on request. Often, owing to time constraints, this can lead to antibiotic prescribing 'over the phone', which one GP referred to as prescribing for 'doctor reasons rather than patient reasons or bacteriological reasons' (GP6). These challenges are all explicitly linked to an increase in antibiotic prescribing by many participants.

I think most of them end up getting an antibiotic to treat as a caution even though maybe it is not as indicated as it would be in the community. (GP2)

You sometimes feel that you are prescribing in those situations without a very definite bug or infection. (GP6)

Occasionally if it is symptomatic UTI you may prescribe over the phone and see how they go. If it is not responding then you obviously need to go see them. (GP5)

Owing to the plethora of clinical issues for discussion at the clinical multidisciplinary team meetings, antimicrobial stewardship was not reported as a key item on the agenda. There are numerous other competing demands on time during the doctor's visit to the LTCF and during the clinical meetings. Participants reported that the regulation of LTCFs by the Health Information and Quality Authority (HIQA) ensures that medication management procedures and pharmacist medication reviews are implemented. It was implied that antibiotic 
audits are only ever conducted to fulfil quality improvement requirements rather than to influence clinical practice. The organisational culture within LTCFs, however, impedes many extra clinical and quality improvement activities because time, and perhaps motivation, is not available.

at the moment there is a linked up thinking between the nursing home and the pharmacy...the triangle isn't complete yet where the GP is involved...there are some GPs who would be going to clinical meetings and having clinical meetings, others wouldn't be. (P7)

since HIQA have leant on them a small bit it is far more detailed, there is a far more joined up thinking between the pharmacy and the home and we have established a set of I suppose a complete medicine management system. (P7)

Then I suppose all it needs is someone like HIQA or the ICGP or the pharmacy crowd to come and say 'look lads you are not practicing properly unless you are doing this' then GPs do adhere to it, they will certainly adhere to it if they are told it is best practice and they all try to adhere to best practice. (GP2) (ICGP: Irish College of General Practitioners)

The domains 'Environmental context and resources' and 'Beliefs about Consequences' are closely aligned. The potential harm or hospitalisation of a vulnerable, comorbid LTCF patient if an antibiotic is not prescribed is a matter of concern to doctors and nurses. The general consensus was that over-treatment with antibiotics and subsequent care in the LTCF is preferred and that hospitalisation should be avoided if at all possible. The domain 'Emotion' is relevant here as participants spoke about fear of the patient coming to harm because of their decision.

...if that means you prescribe the odd antibiotic excessively, I think for the resident most times it's a better scenario for the individual than ending up in an A\&E department because of an untreated infection. It's a balancing act really. (GP1)

...you say look we will hold off on the antibiotic and I have certainly been caught once with a patient who then developed pyelonephritis and was sick and so that learns you alright. (GP7)

There is a lack of acknowledgement that antibiotic prescribing in LTCFs contributes to the public health problem of AMR. Many references to AMR associated it with antibiotic prophylaxis and that it was not common among the patients in the LTCF. There was little discussion about how to avoid the development of AMR and a sense of inevitability regarding this unavoidable problem was evident.

..writing a prescription for an antibiotic is seen as an action or a response, a quick action or a quick response to some problem... I would doubt that resistance is at the forefront of that decision at that time. (C3)

\section{Memory, attention and decision processes}

The variability and complexity of the decision-making process is evident by the findings attributed to the aforementioned TDF domains. For many Doctors, this decision-making process is a culmination of the factors already outlined resulting in a 'balancing act' as they make a risk-benefit assessment of the patient's need for an antibiotic. The fear of the consequences for the patient and the uncertainty around the diagnosis of infection in LTCF patients was clearly linked to the overprescribing of antibiotics in LTCFs by many participants. Much discussion centred on the decision of whether to prescribe an antibiotic or not, with much less thought being given to the decision around which antibiotic to prescribe.

I think if you wait and if the person gets sicker you are kind of damned and if you give them an antibiotic and they really did not have an infection and something else happens to them you are damned. (C1)

you probably do end up prescribing more for the elderly than you would for you or me who are younger, in the fact that you are always slightly worried that if you don' $t$ prescribe then they will get worse. (GP8)

The decision-making autonomy and individual patient care approach dominates the decision-making process for doctors and nurses. Their attention is focused on the patient's clinical presentation, medical history and in some cases the overall care plan. As outlined in 'Beliefs about consequences', the public health threat of AMR does not influence this decision.

I would look to see do they have a temperature, not all the elderly will develop a temperature, some of them are immuno-compromised for various reasons so they don't always necessarily have a temperature. So looking at sats, looking at clinical findings, looking at have they gone off food, are they obviously unwell in themselves. I think that is one thing that sometimes guidelines don't capture. They don't capture that sort of, they will have criteria set down but they don't cover that sort of knowing the patient bit. (GP1)

Several participants acknowledged the valuable support of guidelines to help clinical decision-making. Nurses and pharmacists reported that guidelines are an effective way to ensure that all healthcare professionals were practising evidence-based medicine and that they are a necessity when dealing with outbreaks of infection.

I think there needs to be clear guidelines and protocols in each setting regarding antibiotic use. I do tend to think that there is just generic broad spectrum prescribing of different types of infections without actually doing any sensitivity testing. (P2)

This leads to the important domain of 'Behavioural regulation'. The extent of self-monitoring by means of 
antibiotic surveillance or audit is low and any reported activities generally consisted of participation in the HALT point prevalence studies in some, but not all, of the LTCFs. Participants from all groups welcomed the idea of surveillance and reviewing antibiotic prescribing practices and felt that this would contribute to improving patient care. Doctors were somewhat cautious and several expressed doubt about conducting audits which judge an antibiotic prescription as being appropriate or not. Benchmarking audit results with other centres was not viewed as being a particularly useful exercise by many doctors.

Comparing to other centres, yes but so what? What you are going to do is compare your errors really to their errors. What you need to do is to compare to what you should be doing and see if that can be implemented, if you can do that. (GP6)

A few participants made suggestions for information technology solutions such as decision support systems to guide decision-making but added that a lack of resources would rule out that option.

So I suppose the first thing is the guidance is there, the second thing then is education around the guidance and then you have got to audit it. So it is a cycle, you know the cycle, but whether the government is willing to actually you know follow through with that, that is the big problem, with that is that some investment needs to be made in the education and then people can use it as an audit for their continuing medical education and their medical counsel requirements. (GP1)

\section{APPLICATION OF BCT TAXONOMY AND IDENTIFICATION OF POTENTIAL INTERVENTION FUNCTIONS}

The BCT taxonomy (V.1) has been developed in order to improve the design and implementation of interventions. ${ }^{14}$ In the COM-B model, capability, motivation and opportunity interact to generate behaviour. ${ }^{11}$ Capability represents an individual's physical and psychological ability to undertake an activity. Opportunity represents all the factors outside the individual, that prompt behaviour or make it possible. Motivation involves the brain processes, automatic and reflective, that direct behaviour. The principles of the COM-B model have been applied to the findings of this study to recommend strategies for antimicrobial stewardship in LTCFs as outlined in table 4. Detailed taxonomy has been applied in order to guide the standardisation of the intervention content design and reporting. ${ }^{11}$ The key strategies are: setting goals, education, audit, feedback and monitoring. These strategies have been selected based on the APEASE criteria (Affordable, Practical, Effective/ cost-effective, Acceptable, Safe and Equitable). ${ }^{11}$ Many of the intervention functions were suggested by or discussed with the study participants, thereby improving the likelihood of acceptability in the future. If monitoring and feedback of antibiotic prescribing was introduced, it is possible that comparing or benchmarking the results to other LTCF would motivate healthcare professionals to reflect on and change their prescribing patterns. The TDF domains goals and intentions, which were not represented in the study findings, have been included because clear targets for antimicrobial stewardship are required to motivate behaviour change. Financial incentivisation is suggested but is not likely to be a realistic option as a change to Irish healthcare policy would be required.

\section{DISCUSSION}

This is one of the first studies to investigate the views of healthcare professionals in LTCFs about antibiotic prescribing and to use a behavioural change theory to analyse the findings and suggest intervention strategies for antimicrobial stewardship. The findings have provided valuable information to understand the LTCF antibiotic prescribing culture in great detail. The challenges relating to antimicrobial prescribing in LTCFs were identified along with many broad issues at play such as the organisational culture of LTCFs and healthcare delivery in LTCFs. This study has found that the antibiotic prescribing process is complicated in LTCFs and influenced by social, cultural and contextual issues. The TDF has proven to be a very useful tool for the analysis of the interview findings, in order to encompass the factors influencing the prescribing of antibiotics. Previous qualitative studies of antibiotic prescribing in LTCFs identified the challenges of diagnosing infection in LTCFs, the social pressures from family and nurses, and the variation in practice between different healthcare professionals, without investigating the findings from a theoretical perspective. ${ }^{16} 17$ This study contributes to the knowledge base by providing more evidence to support the importance of behavioural regulation as a strategy for antimicrobial stewardship. The application of the findings to the COM-B model and the BCT taxonomy has provided suggestions for appropriate intervention functions on which to model future antimicrobial stewardship interventions. The results indicate that several intervention functions, such as education around guidelines, audit and feedback to measure performance, and guidance and persuasion by experts in the field, would target the domains identified by the TDF. When the main findings are distilled, the 'behavioural diagnosis' of the relevant COM-B components finds that a key driver for change and antimicrobial stewardship in LTCFs is motivation. It is evident from the findings that antibiotic prescribing in LTCFs is influenced by social and environmental challenges rather than by antimicrobial stewardship results and strategies. In order to raise antimicrobial stewardship as a priority item for patient care and quality improvement, all doctors, nurses and pharmacists involved in LTCFs need to be motivated to reflect on current practice by undertaking antibiotic surveillance in the LTCFs. 


\begin{tabular}{|c|c|c|c|c|}
\hline TDF domain & COM-B & BCT taxonomy & BCT label & $\begin{array}{l}\text { Strategy examples (with intervention } \\
\text { function in italics) }\end{array}$ \\
\hline $\begin{array}{l}\text { Behavioural regulation } \\
\text { Goals. Intentions } \\
\text { Social/professional roles and identity }\end{array}$ & $\begin{array}{l}\text { C-(Psych.) } \\
\text { M-(Refl.) }\end{array}$ & Goals and Planning & $\begin{array}{l}\text { Goal setting } \\
\text { (outcome). } \\
\text { Action planning. } \\
\text { Review outcome } \\
\text { goals }\end{array}$ & $\begin{array}{l}\text { Enablement. Set targets for antibiotic usage } \\
\text { Use antibiotic 'Care Bundles' }\end{array}$ \\
\hline $\begin{array}{l}\text { Knowledge. Memory, attention and } \\
\text { decision-making processes. Behavioural } \\
\text { Regulation } \\
\text { Beliefs about capabilities } \\
\text { Optimism }\end{array}$ & $\begin{array}{l}\text { C-(Psych.) } \\
\text { C-(Phys.) } \\
\text { M-(Refl.) }\end{array}$ & $\begin{array}{l}\text { Shaping knowledge, } \\
\text { Natural consequences, } \\
\text { Comparison of outcomes }\end{array}$ & $\begin{array}{l}\text { Instructions on how to } \\
\text { perform behaviour. } \\
\text { Information about } \\
\text { health consequences. } \\
\text { Credible source }\end{array}$ & $\begin{array}{l}\text { Education: information about antibiotics, } \\
\text { guidelines and AMR } \\
\text { Persuasion: Present information to emphasise } \\
\text { importance of not prescribing antibiotics } \\
\text { inappropriately. Persuasive communication of } \\
\text { information, supported by consultant } \\
\text { microbiologists and geriatricians }\end{array}$ \\
\hline $\begin{array}{l}\text { Environmental context } \\
\text { Memory, attention and decision-making processes }\end{array}$ & $\begin{array}{l}\text { O-(Phys.) } \\
\text { C-(Psych.) } \\
\text { C-(Phys.) }\end{array}$ & $\begin{array}{l}\text { Antecedents, } \\
\text { Associations }\end{array}$ & $\begin{array}{l}\text { Restructuring the } \\
\text { physical environment. } \\
\text { Prompts/cues. } \\
\text { Adding objects to the } \\
\text { environment }\end{array}$ & $\begin{array}{l}\text { Environmental restructure/enablement. Reduce/ } \\
\text { remove LTCF stock of non-first-line antibiotics } \\
\text { (Restriction). } \\
\text { Provide copies of the guidelines and supporting } \\
\text { evidence. } \\
\text { Use antibiotic 'Care Bundles' }\end{array}$ \\
\hline $\begin{array}{l}\text { Knowledge. Memory, attention and } \\
\text { decision-making processes. Behavioural } \\
\text { Regulation }\end{array}$ & $\begin{array}{l}\text { C-(Phys.) } \\
\text { C-(Psych.) } \\
\text { M-(Auto.) }\end{array}$ & Repetition and substitution & $\begin{array}{l}\text { Behavioural practice/ } \\
\text { rehearsal }\end{array}$ & $\begin{array}{l}\text { Training: Practise referring to the guidelines in } \\
\text { daily practice }\end{array}$ \\
\hline Social influences & O-(Soc.) & Social support & $\begin{array}{l}\text { Social support } \\
\text { (practical) }\end{array}$ & $\begin{array}{l}\text { Persuasion and Enablement. Encourage doctors, } \\
\text { nurses and pharmacists to promote guideline } \\
\text { and 'Care Bundle' implementation }\end{array}$ \\
\hline $\begin{array}{l}\text { Goals. Beliefs about Consequences and } \\
\text { Capabilities. Memory, attention and } \\
\text { decision-making processes. Behavioural } \\
\text { Regulation. Social/professional roles and identity. } \\
\text { Social influences }\end{array}$ & $\begin{array}{l}\text { M-(Refl.) } \\
\text { C- (Psych.) } \\
\text { O-(Soc.) }\end{array}$ & $\begin{array}{l}\text { Feedback and Monitoring, } \\
\text { Comparison of outcomes, } \\
\text { Identity }\end{array}$ & $\begin{array}{l}\text { Feedback on outcome } \\
\text { of behaviour } \\
\text { Discrepancy between } \\
\text { current behaviour and } \\
\text { goal } \\
\text { Incompatible beliefs. } \\
\text { Information about } \\
\text { others' approval } \\
\text { Social comparison }\end{array}$ & $\begin{array}{l}\text { Persuasion: Audit and feedback of antibiotic } \\
\text { prescribing and 'Care Bundles' } \\
\text { Enablement: Outline deviations from guidelines/ } \\
\text { evidence-based practice } \\
\text { Persuasion: Benchmark antibiotic usage against } \\
\text { other LTCFs. Consultant review of antibiotic } \\
\text { prescribing }\end{array}$ \\
\hline $\begin{array}{l}\text { Reinforcement. Knowledge. } \\
\text { Beliefs about Capabilities. } \\
\text { Social/professional roles and identity }\end{array}$ & $\begin{array}{l}\text { C-(Psych.) } \\
\text { M-(Refl.) }\end{array}$ & $\begin{array}{l}\text { Reward and threat, } \\
\text { Scheduled consequences }\end{array}$ & $\begin{array}{l}\text { Knowledge } \\
\text { Incentive (outcome) } \\
\text { Reward } \\
\text { approximation/ } \\
\text { completion }\end{array}$ & $\begin{array}{l}\text { Incentivisation: Positive reinforcement from } \\
\text { consultants of audit results } \\
\text { Financial incentive will be provided if antibiotic } \\
\text { prescribing targets met/'care bundles' } \\
\text { implemented }\end{array}$ \\
\hline
\end{tabular}


An important finding of this study is that suboptimal or inappropriate antibiotic prescribing is not something the LTCF participants believed was happening in their LTCF. In similar studies with hospital doctors, suboptimal antibiotic prescribing has been admitted openly and is almost accepted as an inevitable outcome of patient care. ${ }^{27}$ In the hospital setting, suboptimal antibiotic prescribing was accounted for by doctors' benevolence, unwillingness to challenge the hospital medical hierarchy and a coping mechanism for time pressures. ${ }^{27} 28$ This study found that most LTCF healthcare professionals reported satisfactory practices but were not able to support these beliefs with facts as no surveillance activities were in place. This reinforces the need for ongoing behavioural regulation measures in LTCFs, as is conducted in most hospital settings. Antibiotic stewardship strategies are commonly classified as persuasive (education, audit and feedback) or restrictive (restricted formulary, prior authorisation) or structural (eg, computer decision support systems) ${ }^{29}$ A systematic review of interventions to improve antibiotic prescribing in hospitals recommends that both groups of techniques improve patient outcomes and reduce AMR, but that restrictive techniques should only be used when urgent. ${ }^{29}$ In ambulatory care, multifaceted interventions involving educational techniques work best when local barriers to change are addressed. ${ }^{30}$ A systematic review of trials to improve antibiotic prescribing in LTCFs found that educational sessions and material, involving local consensus with staff, are generally acceptable, but the results of most studies were modest and not sustained. ${ }^{31}$ This suggests a greater need to investigate the behavioural reasons to explain these trial results and the use of intervention functions which sustain motivation for change.

The challenge of designing and delivering antimicrobial stewardship interventions in LTCFs may be compounded by the unique organisational culture present, which is different from the hospital and primary setting. It has been well acknowledged that LTCFs have a wide variety of organisational models and service delivery structures, for example, nurse to resident ratio, access to doctor, access to diagnostic equipment or microbiology results. ${ }^{32}$ The influential role of nurses, the variability in practices between LTCFs, and the ethical considerations of caring for patients with dementia and at the end of their life are all characteristic features of LTCF services that must be considered when planning quality improvement strategies. ${ }^{8}$ The Schein model of organisational culture, as previously discussed by Hughes $e t a l^{33}$, suggests that in order to truly understand an organization, a deeper knowledge of the underlying assumptions needs to be analysed, and not just the observable patterns of behaviour. In order to overcome the potential 'normalisation of substandard prescribing practices', the discrepancy between participants' assumptions and reality needs to be addressed. This is important in relation to AMR as participants do not link the public health problem with their LTCF patients, and in relation to antibiotic prescribing, which many assume to be satisfactory in their LTCF without any supporting evidence.

Broom $e t a l^{27}$ have examined antibiotic prescribing decisions in Australian hospital doctors by using Bourdieu's theory of practice to try to understand the disjunction between AMR and suboptimal antibiotic prescribing practices by Doctors. They found that doctors feel a sense of benevolence to their individual patient, which often leads to their over-prescribing antibiotics, without consideration of the public consequences of AMR. This echoes findings in this study which highlight the perception that the public health problem of AMR and antibiotic prescribing in LTCF settings are not connected. It is possible to postulate that a lack of awareness of the true severity and scale of AMR in LTCFs is underpinning this disjunction. If this is the case, then up to date access to local AMR patterns in concise and regular bulletins for healthcare professionals will help to inform and motivate prescribing behaviours. This information, coupled with education on recommended guidelines, will address the 'Knowledge' and 'Beliefs about Consequences' identified in the interview findings. External barriers such as lack of time to use guidelines, difficulty in following the format of guidelines, the inertia of previous practice and lack of outcome expectancy must be addressed by these persuasive education initiatives. ${ }^{34}$ The practicalities of interventions in the LTCF setting must be considered and local issues such as the time available for education and healthcare professional participation in antimicrobial stewardship must be addressed. Fundamental to the success of hospital antimicrobial stewardship interventions is the introduction of a multidisciplinary team including consultants, pharmacists and specialist nurses. ${ }^{35}$ This approach should be adopted in the LTCF setting, especially given the already influential role of the nurse and the potential for expanding the pharmacist's clinical role in this area. The pharmacist already has an existing requirement to visit the LTCF and review the patient's medication at least on a three monthly basis. ${ }^{36} 37$ The recently proposed draft update to the HIQA Standards for Residential Care Settings for Older People in Ireland includes Theme 3 'Safe Service' whereby Standard 3.4.7 recommends that antimicrobial medication is given special consideration. ${ }^{38}$ There is potential here for pharmacists to increase their antimicrobial stewardship activities under the umbrella of this new guidance.

A limitation of the study is social desirability, which is particularly common in prescribing research when the participant gives the answer they feel the interviewer wants to hear. As the interviews progressed, however, it became evident that once the participants felt at ease and that their practices were not being judged, they spoke freely and honestly about the challenges of antibiotic prescribing in LTCFs. Their reports that antibiotics are often prescribed unnecessarily is a testament to that. The fact that the interviewer $(\mathrm{AF})$ is a pharmacist 
may have affected the participant dialogue if they felt that their views or knowledge of antibiotics was being tested. However, this did not emerge as a significant issue as the interview data and the opinions expressed were overall very honest and open. A key strength of the study was the interview method which allowed participants to discuss openly their beliefs and views of the antibiotic prescribing process and the performance of others involved in this process. While all participants were from LTCFs in the greater Cork region, the potential limitation of this was overcome by the broad sampling strategy. The broad sample of professionals with a variety of experience, from a range of LTCF funding categories and sizes, increases the likelihood that these findings are a strong representation of the true factors influencing antibiotic prescribing in Irish LTCFs.

\section{CONCLUSION}

This study provides a detailed insight into behavioural factors influencing the antibiotic prescribing process in LTCFs. The incorporation of behavioural theory, such as the TDF and BCT taxonomy, has supported the identification of key factors such as environmental context and knowledge, which are an integral to understanding antibiotic prescribing in LTCFs. The key component which requires attention in future antimicrobial stewardship interventions is motivation, which will result if participants have in-depth knowledge of antibiotic prescribing practices as captured by antibiotic surveillance. The lack of formal antimicrobial stewardship in LTCFs has also been identified and is recommended as an area to address in future intervention studies. This must become a priority for researchers in this field in order to obtain successful results in antimicrobial stewardship initiatives. It is recommended that future intervention studies incorporate behavioural theory, and standardised BCT taxonomy, to achieve detailed feedback from participants on the successes and challenges of antimicrobial stewardship.

Acknowledgements Our thanks to the doctors, nurses and pharmacists who agreed to be interviewed for this study. The support of University College London Centre for Behaviour Change Summer School 2014 is kindly acknowledged.

Contributors AF conceived the study, conducted the interviews and the analysis and wrote the first draft of the manuscript. SB, CB and SC were involved in the analysis and interpretation of the data. All authors read and approved the final manuscript.

Funding This research was funded by the Health Research Board $\mathrm{PHD} / 2007 / 16$.

Competing interests None.

Ethics approval Clinical Research Committee of the Cork Teaching Hospitals.

Provenance and peer review Not commissioned; externally peer reviewed.

Data sharing statement No additional data are available.

Open Access This is an Open Access article distributed in accordance with the Creative Commons Attribution Non Commercial (CC BY-NC 4.0) license, which permits others to distribute, remix, adapt, build upon this work noncommercially, and license their derivative works on different terms, provided the original work is properly cited and the use is non-commercial. See: http:// creativecommons.org/licenses/by-nc/4.0/

\section{REFERENCES}

1. Nicolle LE. Antimicrobial resistance in long-term care facilities. Future Microbiol 2012;7:171-4.

2. Royal College of Physicians in Ireland Policy Group on Healthcare-Associated Infection. Healthcare-associated infections and nursing homes or extended care settings. Royal College of Physicians in Ireland, 2010.

3. European Centre for Disease Prevention and Control. Point prevalence survey of healthcare-associated infections and antimicrobial use in European long-term care facilities. April-May 2013. Stockholm, 2014

4. Nicolle L, Bentley D, Garibaldi R, et al. Antimicrobial use in long term care facilities. Infect Control Hosp Epidemiol 2000;21:537-45.

5. Daneman N, Gruneir A, Bronskill SE. Prolonged antibiotic treatment in long-term care: Role of the prescriber. JAMA Intern Med 2013;173:673-82.

6. Teixeira Rodrigues A, Roque F, Falcao A, et al. Understanding physician antibiotic prescribing behaviour: a systematic review of qualitative studies. Int J Antimicrob Agents 2013;41:203-12..

7. Tonkin-Crine S, Yardley L, Little P. Antibiotic prescribing for acute respiratory tract infections in primary care: a systematic review and meta-ethnography. J Antimicrob Chemother 2011;66:2215-23.

8. Hughes C, Lapane K, Kerse N. Prescribing for older people in nursing homes: challenges for the future. Int J Older People Nurs2011;6:63-70.

9. Colquhoun HL, Brehaut JC, Sales A, et al. A systematic review of the use of theory in randomized controlled trials of audit and feedback. Implement Sci 2013;8:66.

10. Francis JJ, O'Connor D, Curran J. Theories of behaviour change synthesised into a set of theoretical groupings: introducing a thematic series on the theoretical domains framework. Implement Sci 2012;7:35

11. Michie S, Atkins L, West R. The behaviour change wheel: a guide to designing interventions. Great Britain: Silverback Publishing, 2014.

12. Cane J, O'Connor D, Michie S. Validation of the theoretical domains framework for use in behaviour change and implementation research. Implement Sci 2012;7:37.

13. Duncan EM, Francis JJ, Johnston M, et al. Learning curves, taking instructions, and patient safety: using a theoretical domains framework in an interview study to investigate prescribing errors among trainee doctors. Implement Sci 2012;7:86.

14. Michie S, van Stralen MM, West R. The behaviour change wheel: a new method for characterising and designing behaviour change interventions. Implement Sci 2011;6:42.

15. Michie $S$, Richardson $M$, Johnston $M$, et al. The behavior change technique taxonomy (v1) of 93 hierarchically clustered techniques: building an international consensus for the reporting of behavior change interventions. Ann Behav Med 2013;46:81-95.

16. Schweizer AK, Hughes CM, Macauley DC, et al. Managing urinary tract infections in nursing homes: a qualitative assessment. Pharm World Sci 2005;27:159-65.

17. Walker S, McGeer A, Simor AE, et al. Why are antibiotics prescribed for asymptomatic bacteriuria in institutionalized elderly people? A qualitative study of physicians' and nurses' perceptions. CMAJ 2000;163:273-7.

18. Medical Research Council. Developing and evaluating complex interventions: new guidance. Medical Research Council, 2008.

19. Richie J, Lewis J. Qualitative research practice a guide for social science students and researchers. London: Sage Publications, 2008.

20. Sandelowski M. Sample size in qualitative research. Res Nurs Health 1995;18:179-83.

21. Pope C, Ziebland S, Mays N. Qualitative research in health care. Analysing qualitative data. BMJ 2000;320:114-16.

22. QSR International Pty Ltd. NVivo Qualitative Data Analysis Software Version 10.2012.

23. Tong A, Sainsbury. P, Craig. J. Consolidated criteria for reporting qualitative research (COREQ): a 32-item checklist for interviews and focus groups. Int J Qual Health Care 2007;19:349-57.

24. Strategy for Antimicrobial Resistance in Ireland. Guidelines for antimicrobial prescribing in primary care in Ireland. Health Service Executive, Health Protection Surveillance Centre, 2011.

25. Health Protection Surveillance Centre. HPSC Publications. 2013 [cited 9 July 2013]; http://www.hpsc.ie/hpsc/Publications/.

26. Institute for Healthcare Improvement. Raising the bar with bundles: treating patients with an all-or-nothing standard. 2006. http://www.ihi. org/lHI/Topics/CriticalCare/IntensiveCare/Literature/Raisingthe BarwithBundles.htm 
27. Broom A, Broom J, Kirby E. Cultures of resistance? A Bourdieusian analysis of doctors' antibiotic prescribing. Soc Sci Med. 2014;110:81-8.

28. Charani E, Castro-Sanchez E, Sevdalis N, et al. Understanding the determinants of antimicrobial prescribing within hospitals: the role of "prescribing etiquette". Clin Infect Dis 2013;57:188-96.

29. Davey P, Brown E, Charani E, et al. Interventions to improve antibiotic prescribing practices for hospital inpatients. Cochrane Database Syst Rev 2013;4:CD003543.

30. Arnold SR, Straus SE. Interventions to improve antibiotic prescribing practices in ambulatory care. Cochrane Database Syst Rev 2005;(4): CD003539.

31. Fleming A, Browne J, Byrne S. The effect of interventions to reduce potentially inappropriate antibiotic prescribing in long-term care facilities: a systematic review of randomised controlled trials. Drugs Aging 2013;30:401-8.

32. Tjia J, Gurwitz JH, Briesacher BA. Challenge of changing nursing home prescribing culture. Am J Geriatr Pharmacother 2012;10:37-46.
33. Hughes CM, Lapane K, Watson MC, et al. Does organisational culture influence prescribing in care homes for older people? A new direction for research. Drugs Aging 2007;24:81-93.

34. Cabana MD, Rand CS, Powe NR, et al. Why don't physicians follow clinical practice guidelines? A framework for improvement. JAMA 1999;282:1458-65.

35. SARI Hospital antimicrobial stewardship working group. Guidelines for antimicrobial stewardship in hospitals in Ireland 2009. http://www. hpsc.ie/hpsc/A-Z/MicrobiologyAntimicrobialResistance/ InfectionControlandHAI/Guidelines/File,4116,en.pdf

36. Health Information and Quality Authority. National quality standards for residential care settings for older people Ireland. Health Information and Quality Authority, 2008.

37. Pharmaceutical Society of Ireland. Guidance on the supply by Pharmacists in retail pharmacy businesess of medicines to patients in residential care settings/nursing homes. 2014

38. Health Information and Quality Authority. Draft National Standards for Residential Care Settings for Older People in Ireland HIQA. July 2014, Report No. 